\title{
Inhibitors of Class I Histone Deacetylases Reverse Contextual Memory Deficits in a Mouse Model of Alzheimer's Disease
}

\author{
Mark Kilgore ${ }^{1,4}$, Courtney A Miller ${ }^{1,4}$, Daniel M Fass ${ }^{2,3}$, Krista M Hennig ${ }^{2,3}$, Stephen J Haggarty ${ }^{2,3}$, \\ J David Sweatt' and Gavin Rumbaugh*,I \\ 'Department of Neurobiology, Evelyn F McKnight Brain Institute, University of Alabama at Birmingham School of Medicine, Birmingham, AL, USA; \\ ${ }^{2}$ Stanley Center for Psychiatric Research, Broad Institute of Harvard University and Massachusetts Institute of Technology, Cambridge, MA, USA; \\ ${ }^{3}$ Center for Human Genetic Research, Massachusetts General Hospital, Harvard Medical School, Boston, MA, USA
}

\begin{abstract}
Alzheimer's disease (AD) is a neurodegenerative disorder characterized clinically by cognitive impairments that progress to dementia and death. The earliest symptoms of AD present as a relatively pure deficit in memory retrieval. Therefore, drug treatments that intervene in the early stages of AD by rescuing memory deficits could be promising therapies to slow, or even reverse progression of the disease. In this study, we tested the potential of systemic histone deacetylase inhibitor (HDACi) treatment to rescue cognitive deficits in a mouse model of AD. APPswe/PSIdE9 mice showed pronounced contextual memory impairments beginning at 6 months of age. Chronic HDACi injections (2-3 weeks) did not alter contextual memory formation in normal mice, but had profound effects in transgenic animals. Injections of sodium valproate, sodium butyrate, or vorinostat (suberoylanilide hydroxamic acid; Zolinza $\mathbb{R}$ ) completely restored contextual memory in these mutant mice. Further behavioral testing of the HDACi-treated transgenic mice showed that the newly consolidated memories were stably maintained over a 2-week period. Measurement of the HDAC isoform selectivity profile of sodium valproate, sodium butyrate, and vorinostat revealed the common inhibition of class I HDACs (HDACl, 2, 3, 8) with little effect on the class Ila HDAC family members (HDAC4, 5, 7, 9) and inhibition of HDAC6 only by vorinostat. These preclinical results indicate that targeted inhibition of class I HDAC isoforms is a promising avenue for treating the cognitive deficits associated with early stage AD. Neuropsychopharmacology (20 I0) 35, 870-880; doi:I 0.1038/npp.2009.197; published online 9 December 2009
\end{abstract}

Keywords: Alzheimer's disease; cognition; drug discovery; epigenetics; histone deacetylase inhibitor; fear memory

\section{INTRODUCTION}

Alzheimer's disease (AD) is characterized by debilitating memory impairments and significant neural degeneration. The earliest stages of $\mathrm{AD}$ are characterized by a relatively pure impairment of episodic memory (American Psychiatric Association, Task Force on DSM-IV, 1994). As the disease progresses, neurodegeneration in the temporal and frontal lobes becomes more profound and symptoms worsen (Braak et al, 1998b). These memory impairments result in a poor quality of life for the patient and hardship for the patient's family. As a result, developing therapeutic strategies to enhance the encoding, maintenance, and retrieval of memories is critical for improved $\mathrm{AD}$ patient outcomes.

* Correspondence: Dr G Rumbaugh, Department of Neurobiology, Evelyn F McKnight Brain Institute, University of Alabama at Birmingham, 972 Shelby Building, 1825 University Blvd, Birmingham, AL 35294, USA, Tel: + | 205996 64 I2, Fax: + I 2059755097 ,

E-mail: grumbaugh@nrc.uab.edu

${ }^{4}$ These authors contributed equally to this work.

Received 21 May 2009; revised 7 October 2009; accepted 26 October 2009
The brains of $\mathrm{AD}$ patients have extensive extracellular $\beta$-amyloid $(\mathrm{A} \beta)$ plaques and intracellular neurofibrillary tangles (Braak and Braak, 1998a; Dickson, 1997). The extracellular plaques are formed through deposits of insoluble A $\beta$ aggregates secreted from neurons. Recently, several studies have shown that soluble oligomers of $\mathrm{A} \beta$ are sufficient to cause structural and functional changes to neurons (Haass and Selkoe, 2007; Walsh et al, 2002). As a result, elevated levels of $\mathrm{A} \beta$ are believed to contribute to the cognitive impairments associated with $\mathrm{AD}$ (Lambert et al, 1998). A certain fraction of AD cases are early onset, and arise through autosomal inheritance of one of several causative genetic mutations (Selkoe, 2001). Several transgenic mouse lines have been developed that model the amyloidosis thought to underlie the onset of familial $\mathrm{AD}$ (Gotz et al, 2004; Wong et al, 2002). Overexpression of these human mutations in mice induces amyloidosis and these animals have significant cognitive impairments. In particular, mice that overexpress the delta exon 9 variant of presenilin-1 (PS1), in combination with the Swedish mutation of amyloid precursor protein (APP), have considerable levels of the aggregating form of $\mathrm{A} \beta, \mathrm{A} \beta-42$ (Jankowsky et al, 2004). Plaque deposition in this line is very aggressive 
and occurs as early as 6 months of age (Jankowsky et al, 2004), though cognitive impairments are not detected until much later in the cogenic line (Savonenko et al, 2005; but see also Jankowsky et al, 2005). Therefore, if early memory deficits were uncovered in this mouse line, it could serve as a particularly useful model to study early stage memory impairments associated with $\mathrm{AD}$.

Episodic memory is severely disrupted in patients with $\mathrm{AD}$. Studies over the past 50 years have shown that the medial temporal lobe, particularly the hippocampal formation and associated cortical areas, are directly involved in the encoding and consolidation of these memories (Eichenbaum, 2000; Squire et al, 2004). Associative learning activates biochemical signaling pathways that trigger the synthesis of new proteins necessary for the consolidation of hippocampus-dependent memories (Kandel, 2001; McGaugh, 2000). Because memory consolidation in the hippocampus is particularly susceptible to dysfunction during the early stages of $\mathrm{AD}$, we hypothesized that the molecular mechanisms required for the synthesis of memory-promoting proteins are dysfunctional in $\mathrm{AD}$. Consistent with this idea, pharmacologically stimulating gene transcription regulators that participate in memory formation may enhance cognitive performance in patients with this disease.

Histone acetyltransferases (HATs) catalyze the acetylation of lysine residues on core histone proteins, relaxing chromatin structure and facilitating gene transcription (Berger, 2007; Jenuwein and Allis, 2001; Kouzarides, 2007). This post-translational modification is reversed by histone deacetylases (HDACs), which remove the acetyl groups, promoting compaction of chromatin and a reduction in the transcription of corresponding genes. Histone acetylation occurs in the hippocampus in response to contextual learning (Levenson et al, 2004), and reducing the levels of histone acetylation by preventing HAT activity promotes amnesia and interferes with the consolidation of hippocampus-dependent memories (Alarcon et al, 2004; Korzus et al, 2004; Wood et al, 2006). In contrast, inhibiting HDACs, which artificially increases the acetylation and transcription of some genes, enhances hippocampusdependent memory formation (Guan et al, 2009; Levenson et al, 2004; Vecsey et al, 2007; Wood et al, 2006). Recently, inhibitors of histone deacetylases (HDACi's) have been shown to restore cognitive function in an inducible model of neurodegeneration (Fischer et al, 2007). Therefore, we investigated the potential for HDACi treatment to restore memory consolidation and facilitate the storage of information in a mouse model of $\mathrm{AD}$.

\section{MATERIALS AND METHODS}

\section{Animals}

All mice used in this study were APPswe/PS1dE9 doubletransgenic mice (APP/PS1) originally described as Line 85 (Jankowsky et al, 2004), and were obtained from the Jackson Laboratory (Bar Harbor, ME) with strain name B6C3-Tg (APPswe,PSEN1dE9)85Dbo/J; stock number 004462). These mice express a chimeric mouse/human APP transgene that contains the Swedish mutations (K595N/M596L) as well as a mutant human PS1 transgene carrying the deleted exon 9 variant under control of mouse prion promoter elements. The two transgenes co-segregate in this mouse line (Jankowsky et al, 2004). Mice were maintained as double hemizygotes by crossing transgenepositive males with wild-type (WT) females on a B6C3F1/J background strain (Jackson Laboratories: stock number 100010). Similar numbers of male and female subjects were used in each experiment. All mice were genotyped through tail clips and subsequent PCR analysis of genomic DNA. Mice were housed under 12:12 light/dark cycles, with food and water available ad libidum. All procedures were performed in accordance with the University of Alabama at Birmingham Institutional Animal Care and Use Committee and with national regulations and policies.

\section{Drugs}

Sodium valproate (VPA; Sigma; P-4543) was dissolved in $0.1 \mathrm{M}$ PBS and mice were given $100 \mathrm{mg} / \mathrm{kg}$ doses. Sodium butyrate (NaB; Sigma; 19364) was dissolved in Milli-Q water and mice received $1.2 \mathrm{~g} / \mathrm{kg}$ doses. Suberoylanilide hydroxamic acid (SAHA) was synthesized by Medicilon/MPI Preclinical Research as described previously in WO 93/ 07148 PTC/US92/08454 (Gediya et al, 2005) and was given to mice as $50 \mathrm{mg} / \mathrm{kg}$ doses. SAHA was dissolved in $100 \%$ DMSO at a concentration of $50 \mathrm{mg} / \mathrm{ml}$ and then further diluted to $5 \mathrm{mg} / \mathrm{ml}$ in $0.9 \%$ saline. Injections $(10 \mathrm{ml} / \mathrm{kg})$ were given intraperitoneally for all drugs and were alternated daily between left and right sides of the abdomen.

\section{Behavioral Procedures}

For contextual fear conditioning, mice were placed into the training chamber and allowed to explore for $2.5 \mathrm{~min}$, after which they received an electric footshock $(2 \mathrm{~s}, 0.75 \mathrm{~mA})$. After the shock, the mice remained in the chamber for an additional $28 \mathrm{~s}$ before being returned to their home cage. To test for long-term memory (LTM), all mice were returned to the training chamber $24 \mathrm{~h}$ later, and allowed to explore the context for $5 \mathrm{~min}$. For reexposure experiments, mice were injected for an additional 14 days after training, before a second, $5 \mathrm{~min}$ reexposure to the context. Injections were given $2 \mathrm{~h}$ before training and testing. Activity was determined using an algorithm (Video Freeze; Med Associates) that subtracts differences between successive video frames. Freezing was defined as raw activity counts that fall below a preset threshold for more than $1 \mathrm{~s}$. The algorithm was tested for accuracy by manually scoring at least three randomly chosen videos in each experiment. In all cases, the algorithm performed as accurately as a human observer.

\section{Isolation of Hippocampus and Histone Extraction}

Brains were first immersed in oxygenated $\left(95 \% / 5 \% \mathrm{O}_{2} / \mathrm{CO}_{2}\right)$ ice-cold cutting saline (in mM: 110 sucrose, $60 \mathrm{NaCl}, 3 \mathrm{KCl}$, $1.25 \mathrm{NaH}_{2} \mathrm{PO}_{4}, 28 \mathrm{NaHCO}_{3}, 0.5 \mathrm{CaCl}_{2}, 7 \mathrm{MgCl}_{2}, 5$ glucose, 0.6 ascorbate) immediately after rapid decapitation. The hippocampus was then dissected, frozen on dry ice, and stored at $-80^{\circ} \mathrm{C}$ overnight. For histone extractions, all procedures were performed on ice and all solutions were chilled to $4^{\circ} \mathrm{C}$ before use unless otherwise indicated. All centrifugation steps were performed at $4^{\circ} \mathrm{C}$. Tissue 
homogenates were centrifuged at $7700 \mathrm{~g}$ for $1 \mathrm{~min}$. The supernatant (cytoplasmic fraction) was aspirated and the pellet (nuclear fraction) was resuspended in $1 \mathrm{ml} 0.4 \mathrm{~N}$ $\mathrm{H}_{2} \mathrm{SO}_{4}$. Histones were acid extracted from the nuclear fraction for $30 \mathrm{~min}$, followed by centrifugation at $14000 \mathrm{~g}$ for $10 \mathrm{~min}$. The supernatant was transferred to a fresh tube, and proteins were precipitated with $100 \%$ trichloroacetic acid containing deoxycholic acid (Na+salt; Sigma) for $30 \mathrm{~min}$. Precipitated proteins were collected by centrifugation at $14000 \mathrm{~g}$ for $30 \mathrm{~min}$. The supernatant was discarded and the protein pellet was washed with $1 \mathrm{ml}$ of acidified acetone $(0.1 \% \mathrm{HCl})$ followed by $1 \mathrm{ml}$ acetone, for $5 \mathrm{~min}$ each. Protein precipitates were collected between washes by centrifugation $(14000 \mathrm{~g}, 5 \mathrm{~min})$. The resulting purified proteins were resuspended in $50 \mathrm{mM}$ Tris $(\mathrm{pH}$ 8.0) and stored at $-80^{\circ} \mathrm{C}$.

\section{Western Blotting}

Acetylation levels of histone extracts were assayed by western blotting. Loading buffer was added (final concentration: $6.25 \mathrm{mM}$ Tris (pH 6.8), $2 \%$ SDS, $10 \%$ glycerol, $1.25 \%$ 2-mercaptoethanol, $0.1 \%$ bromophenol blue) and protein extracts $(1 \mu \mathrm{g})$ were separated by SDS-PAGE on a $15 \%$ resolving gel and transferred to PVDF membranes for immunoblotting. Membranes were briefly rinsed with $100 \%$ methanol, air-dried for $15 \mathrm{~min}$, and washed with TBST (in $\mathrm{mM}$ : $150 \mathrm{NaCl}, 20$ Tris (pH 7.5), $0.05 \%$ Tween 20) for $5 \mathrm{~min}$ at room temperature. The membranes were then blocked in $3 \%$ bovine serum albumin (BSA) in TBST for $45 \mathrm{~min}$ at room temperature and then incubated in primary antibody (1:5000 for all primary antibodies used) containing TBST and $5 \% \mathrm{BSA}$ for $1 \mathrm{~h}$ at room temperature. The following anti-rabbit primary antibodies were used: anti-histone $\mathrm{H} 3$, anti-acetyl histone $\mathrm{H} 3$ (Lys-14), anti-histone H4, antiacetyl-H4 (Lys-5/Lys-8/Lys-12/Lys-16) (all supplied by Upstate). This was followed by three washes in TBST and incubation in horseradish-peroxidase-conjugated anti-rabbit secondary antibody $(1: 10000$; Vector Labs) for $45 \mathrm{~min}$ at room temperature. The membranes were again rinsed in TBST and immunolabeling of membranes was detected by chemiluminescence (ECL; Pierce). Films were scanned and images were analyzed by densitometry.

\section{HDAC Biochemical Assays}

The in vitro activities of recombinant human HDACs 1-9 (BPS Biosciences) were measured with a 384-well-platebased fluorometric deacetylase assay making use of acetylated tripeptide substrates that are amide-coupled to 7-amino-4-methylcoumarin that can detect either class I/IIb (substrate MAZ1600) or class IIa/HDAC8 (substrate MAZ1675) HDAC activity as described in detail in Bradner et al (2009), with the following modifications: HDAC1 (4.5 ng per reaction; MAZ1600 $K_{\mathrm{m}}=6 \mu \mathrm{M}$ ); HDAC2 (4 ng per reaction; MAZ1600 $K_{\mathrm{m}}=4.5 \mu \mathrm{M}$ ); HDAC3 (2 ng per reaction; MAZ1600 $\left.K_{\mathrm{m}}=9.5 \mu \mathrm{M}\right)$; HDAC4 $(0.1 \mathrm{ng}$ per reaction; MAZ1675 $K_{\mathrm{m}}=8 \mu \mathrm{M}$ ); HDAC5 (1 ng per reaction; MAZ1675 $\left.K_{\mathrm{m}}=57 \mu \mathrm{M}\right) ; \quad$ HDAC6 (50 ng per reaction; MAZ1600 $\left.K_{\mathrm{m}}=7 \mu \mathrm{M}\right)$; HDAC7 $\left(0.3 \mathrm{ng}\right.$ per reaction; MAZ1675 $K_{\mathrm{m}}=$ $20 \mu \mathrm{M}$ ); HDAC8 (3 ng per reaction; MAZ1675 $K_{\mathrm{m}}=530 \mu \mathrm{M}$ ); HDAC9 (1.5 ng pre reaction; MAZ1675 $K_{\mathrm{m}}=37 \mu \mathrm{M}$ ); TCEP was omitted from the assay buffer. Rates of reactions (slopes) were normalized to the mean of DMSO control treatments for each enzyme on each plate.

\section{Statistical Analysis}

One- or two-way univariate or repeated-measures analysis of variance was applied to all data. Details on the specific statistical model used in each experiment can be found in the figure legends. Student's $t$-tests and the Scheffe's post hoc test were used when necessary. Significance was set at $P \leqslant 0.05$ for all tests.

\section{RESULTS}

To test the hypothesis that systemic injections of HDACi's are able to rescue early cognitive deficits in an AD model, we first performed a series of experiments to establish when hippocampus-dependent memory deficits first appear in APP/PS1 mice. We chose to use contextual fear conditioning as a memory assay for two reasons. This associative learning paradigm is hippocampus dependent (Phillips and LeDoux, 1992), requires only a single trial, and results in a robust, long-lasting memory for a context. Thus, fear conditioning allows for the analysis of multiple stages of memory in a brain region thought to be severely affected in early stage AD. WT and aged-matched double-transgenic mice were trained at either 4 or 6 months of age with a mild training paradigm. The specific protocol was designed to yield approximately $40-50 \%$ freezing in a retrieval test, enabling us to detect both enhancements and deficits in memory strength. Transgenic mice (4-month old) showed levels of freezing identical to aged-matched WT littermates during the LTM test (Figure 1a; $\mathrm{F}_{(1,21)}=0.07, P>0.05$ ). In contrast, a pronounced 24-h memory deficit was observed in 6-month-old transgenic mice $\left(\mathrm{F}_{(1,12)}=6.53, P<0.05\right)$, indicating that contextual memory deficits are present in these mice as early as 6 months of age. Importantly, there were no differences between WT and APP/PS1 mice in the amount of exploratory activity during the $150 \mathrm{~s}$ period before the footshock at the time of training (4-month-old WT: $147.2 \pm 19.9, \quad$ APP/PS1: $129.2 \pm 18.8, \quad \mathrm{~F}_{(1,20)}=0.43$, $P>0.05$; 6-month-old WT: $42.6 \pm 8.4$, APP/PS1: $57.2 \pm 15.2$, $\left.\mathrm{F}_{(1,12)}=0.64, P>0.05\right)$. Nor were there differences in the amount of post-shock freezing during training (4-monthold WT: $23.7 \pm 5.5$, APP/PS1: $21.4 \pm 5.3, \quad \mathrm{~F}_{(1,20)}=0.10$, $P>0.05$; 6-month-old WT: $31.4 \pm 8.3$, APP/PS1: $31.8 \pm 11.5$, $\left.\mathrm{F}_{(1,12)}=0.001, P>0.05\right)$. Given the memory deficit in the 6-month-old transgenic mice, we next tested their ability to acquire context-shock associations. WT and transgenic mice were given a retrieval test $1 \mathrm{~h}$ after training. Interestingly, APP/PS1 mice had normal freezing at this time point (Figure $1 \mathrm{~b} ; \mathrm{F}_{(1,13)}=0.17, P>0.05$ ). This intact short-term memory indicates that APP/PS1 mice are able to acquire contextual associations successfully at this age. Taken together, these data indicate that 6-month-old APP/PS1 mice have deficits in memory formation arising from abnormal consolidation and/or retrieval processes.

We next tested the possibility that HDACi's ameliorate the memory deficits in these mice. WT and APP/PS1 mice were chronically treated with either vehicle or valproic acid 
before training with the mild fear conditioning protocol. At $24 \mathrm{~h}$ after training, all mice were given a 5 min retrieval test. Similar to our finding (see Figure 1 ), we detected an interaction between genotypes $\left(\mathrm{F}_{(3,23)}=19.10, P<0.001\right)$.

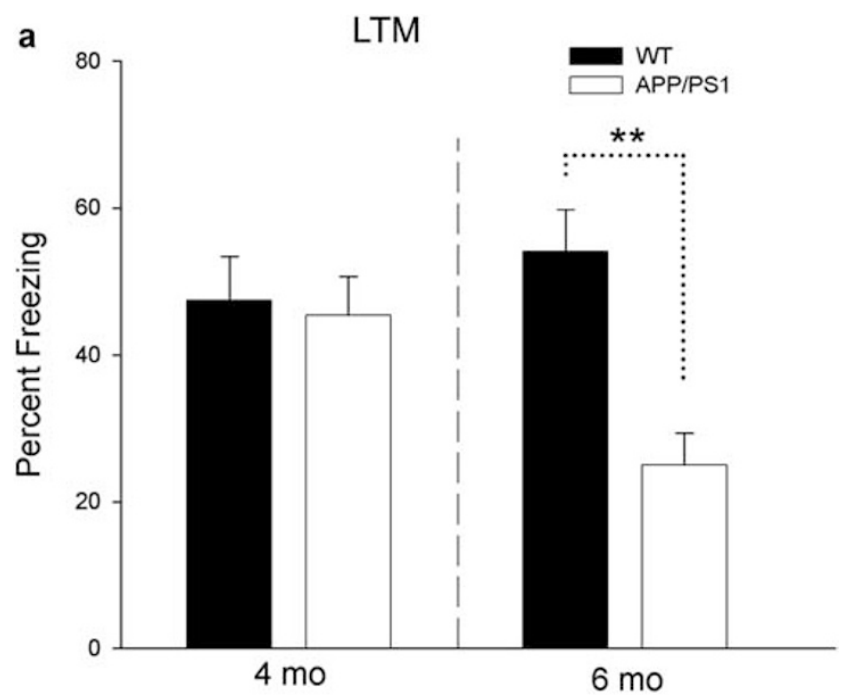

b

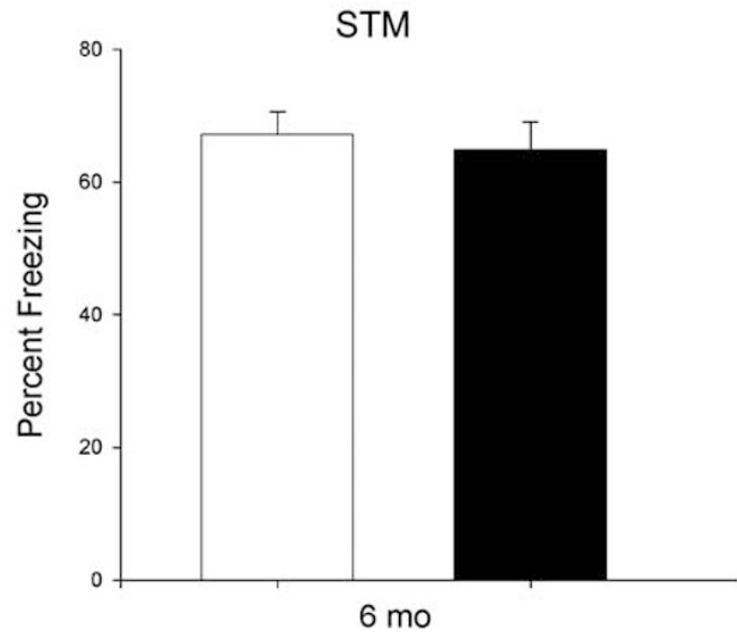

Figure I APPswe/PSIdE9 mice develop contextual memory deficits with age. (a) Wild-type (WT) and APPswe/PSI dE9 (4- or 6-month-old) mice were trained in a contextual fear conditioning paradigm (4 months: WT $n=\mid 1$, APP/PS $\mid n=11 ; 6$ months: WT $n=6$, APP/PS $\mid n=7$ ). After $24 \mathrm{~h}$, all mice were reexposed to the conditioning context. Percent freezing was calculated for each animal. Analysis of variance (ANOVA), ${ }^{* *} P<0.01$. (b) WT and APP/PSI (6-month-old) mice were trained in a contextual fear conditioning paradigm (WT $n=7$, APP/PSI $n=7$ ). After I h, all mice were reexposed to the context to assess short-term memory. Percent freezing was calculated for each animal. ANOVA, P>0.05.

Figure 2 Sodium valproate treatment rescues contextual memory formation in APPswe/PSIdE9 mutants. (a) Wild-type (WT) and APP/PSI mice were injected with either vehicle (VEH) or $100 \mathrm{mg} / \mathrm{kg}$ sodium valproate (VPA) daily for 2 weeks (VEH: WT $n=7$, APP/PSI $n=7$; VPA: WT $n=6$, APP/PSI $n=7$ ). All mice were then trained in a contextual fear conditioning paradigm and tested for long-term memory $24 \mathrm{~h}$ later by reexposure to the conditioning context. Freezing was measured for each animal. Two-way univariate analyses with Scheffe's post hoc tests were performed. $* P<0.05$, ** $P<0.0$ I. (b) Exploratory activity was measured for each animal during 150 s period before the footshock. (c) Percent freezing was calculated for each animal during the $28 \mathrm{~s}$ period immediately after the footshock.
Vehicle-treated APP/PS1 mice had significantly lower freezing compared to vehicle-treated WT littermates during the LTM test (Figure 2a; $t_{(12)}=5.69, P<0.005$ ). We also
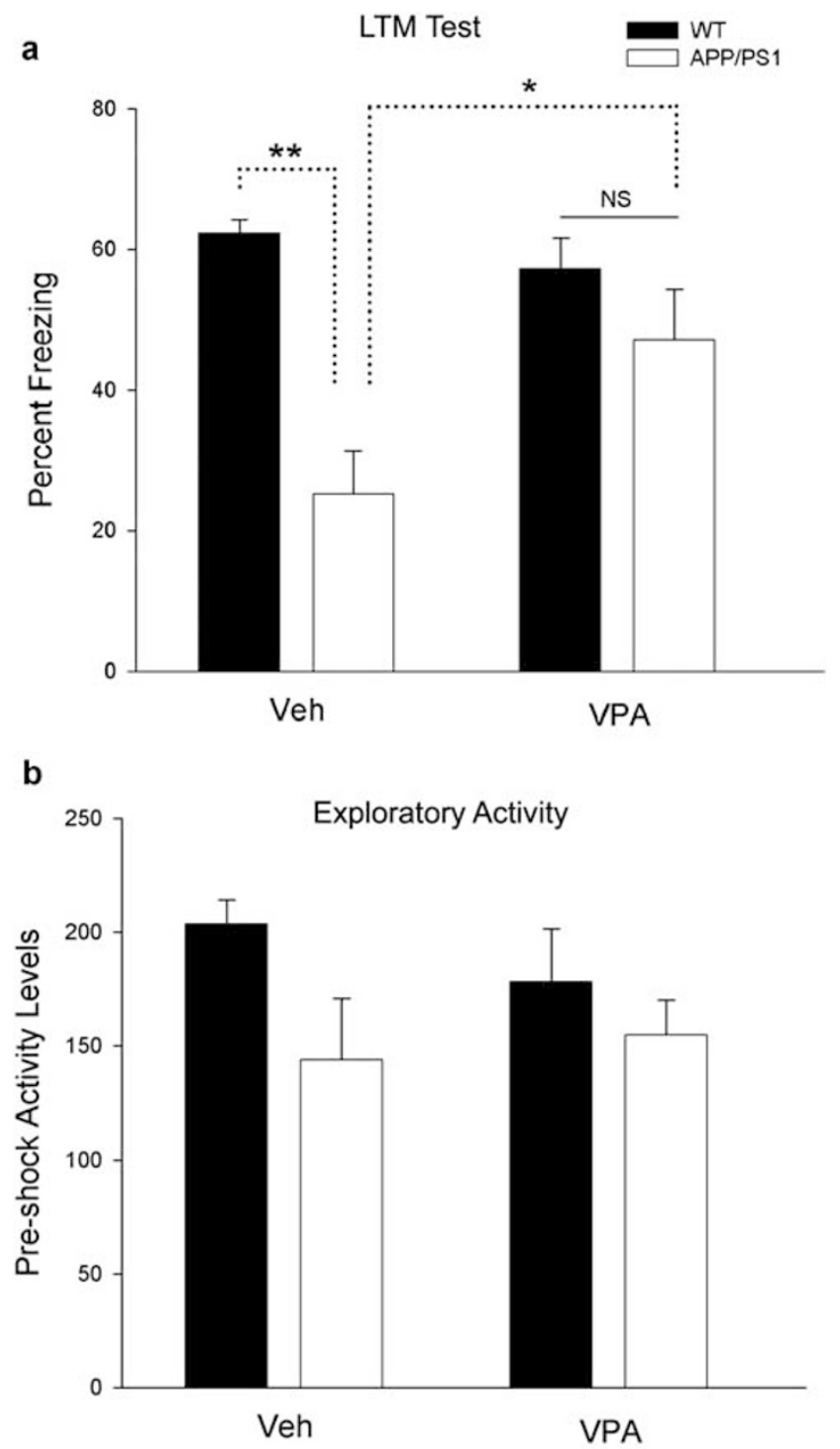

C

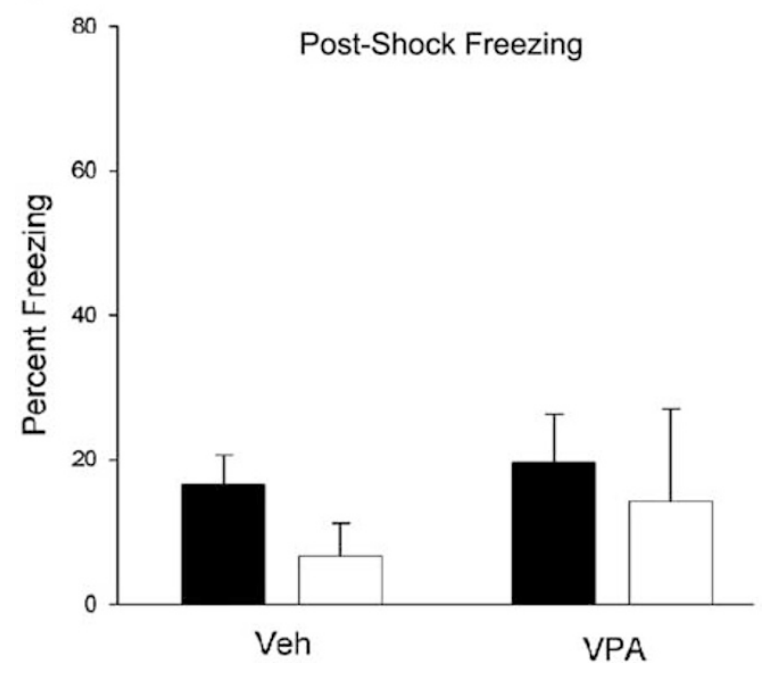


detected a drug by genotype interaction $\left(\mathrm{F}_{(3,23)}=6.32\right.$, $P<0.05)$. VPA treatments did not alter freezing behavior in WT mice $\left(t_{(11)}=1.12, P>0.05\right)$. In contrast, VPA-treated APP/PS1 mice had significantly higher freezing levels during the $24 \mathrm{~h}$ retrieval test when compared to vehicle-treated mutant littermates $\left(\mathrm{t}_{(12)}=-2.33, P<0.05\right.$; Figure $\left.2 \mathrm{a}\right)$. In addition, mutants treated with valproic acid were not significantly different from either WT group, indicating that HDACi treatments restored performance to normal levels for a 6-month-old mouse (Figure 2a; P's $>0.05$ ). We detected no significant differences among groups in exploratory activity or immediate, post-shock freezing during the training session (Figure $2 \mathrm{~b}$ and $\mathrm{c}$; see legend for statistics), indicating that there were no state-dependent effects associated with VPA treatment and that APP/PS1 mice do not differ from WT controls in their behavior during training.

In a separate series of experiments, we tested the hypothesis that other HDACi's would rescue freezing deficits in this $\mathrm{AD}$ model. Indeed, chronic treatments of SAHA (Figure 3a; see legend for statistics) or $\mathrm{NaB}$ (Figure $4 \mathrm{a}$, see legend for statistics) completely restored performance deficits in APP/PS1 mice. We did not detect an effect of $\mathrm{NaB}$ on WT mice. Similar to VPA treatments, these compounds had no effect on exploratory activity or postshock freezing levels (Figures $3 \mathrm{~b}$ and $\mathrm{c}$, and $4 \mathrm{~b}$ and $\mathrm{c}$ ). Taken together, our data strongly suggest that chronically treating APPswe/PS1dE9 double-transgenic mice with broad-spectrum HDACi's rescues freezing deficits by enhancing memory formation.

Contextual memories are initially consolidated in the hippocampus and amygdala, but are eventually stabilized over the course of days to weeks through increasing involvement of neocortical circuits (Dudai, 2004; Frankland and Bontempi, 2005; Medina et al, 2008). Because the mechanisms of memory formation and memory storage are distinct, we were curious if memories formed in HDACitreated APP/PS1 mice successfully stabilized over time. To test this idea, mice from the previous $\mathrm{NaB}$ experiment continued to receive daily injections for an additional 14 days. All mice were again reexposed to the conditioning context and freezing behavior was measured. Overall, results from the 15-day retrieval tests were qualitatively similar to those observed during the $24 \mathrm{~h}$ retrieval test (Figures $4 \mathrm{a}$ and 5a; Genotype: $\mathrm{F}_{(3,34)}=7.92, P<0.01$; Drug: $\left.\mathrm{F}_{(3,34)}=23.45, P>0.001\right)$. Untreated APP/PS1 mice had freezing levels that were statistically different from all other groups (Figure 5a; Veh APP/PS1 vs Veh WT: $t_{(16)}=4.42$, $P<0.001 ;$ Veh APP/PS1 vs $\mathrm{NaB}$ APP/PS1: $t_{(17)}=-4.43$,

Figure 3 Suberoylanilide hydroxamic acid (SAHA) rescues memory deficits in APPswe/PSIdE9 mice. (a) Wild-type (WT) and APP/PSI (6-month-old) mice were injected with either vehicle $(\mathrm{VEH})$ or $50 \mathrm{mg} / \mathrm{kg}$ SAHA daily for 19 days (VEH: WT $n=7$, APP/PSI $n=7$; NaB: APP/PS I $n=7$ ). All mice were then trained in a contextual fear conditioning paradigm and tested for long-term memory $24 \mathrm{~h}$ later. Freezing was measured for each animal. One-way analysis of variance (ANOVA) tests were performed, $F_{(2,20)}=8.93, P<0.005$. $* P<0.05$, *** $P<0.0$ l. (b) Exploratory activity was measured for each animal during $150 \mathrm{~s}$ period before the footshock $\left(F_{(2,18)}=0.06, P>0.05\right)$. (c) Percent freezing was calculated for each animal during the $28 \mathrm{~s}$ period immediately after the footshock $\left(F_{(2,18)}=1.50, P>0.05\right)$.
$P<0.001$ ), whereas freezing performance in the HDACitreated APP/PS1 mice was not significantly different from either WT group (Drug by Genotype: $F_{(3,34)}=2.71$, $P>0.05)$. This indicates that newly consolidated contextual
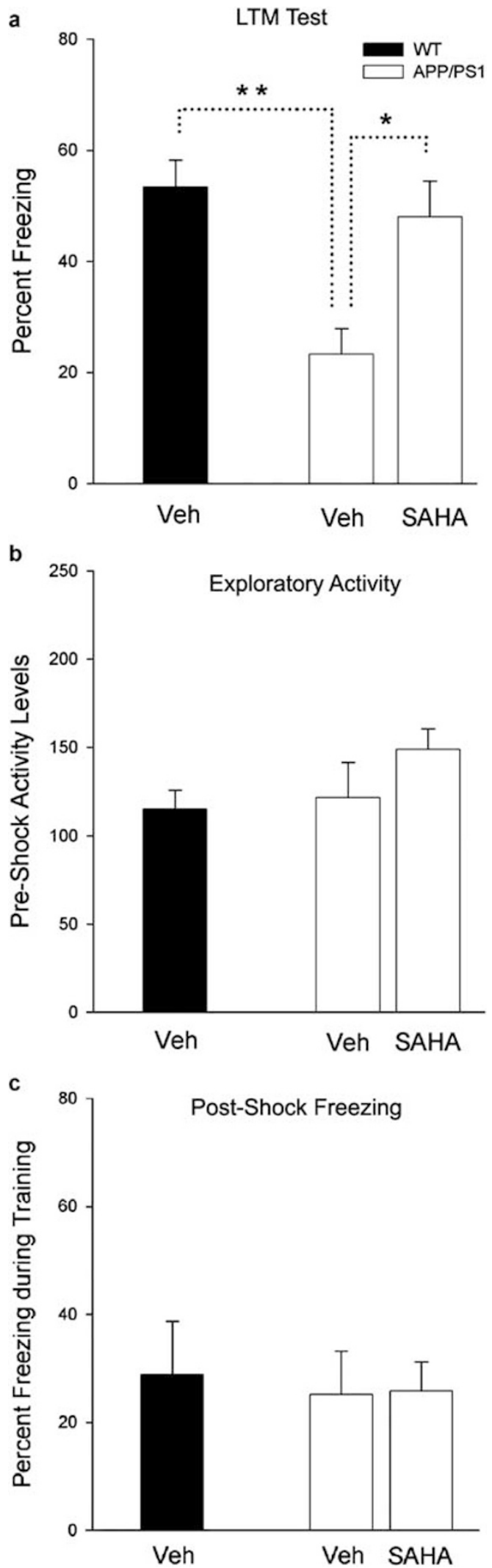
memories successfully achieve stability over time in mutants treated with HDACi's. Interestingly, we also detected a significant difference between WT mice treated with $\mathrm{NaB}$ relative to the WT vehicle group (Figure 5a; $\left.t_{(17)}=-2.35, P<0.05\right)$, accounting for the lack of a drug by genotype interaction. This could be due to an increase

a

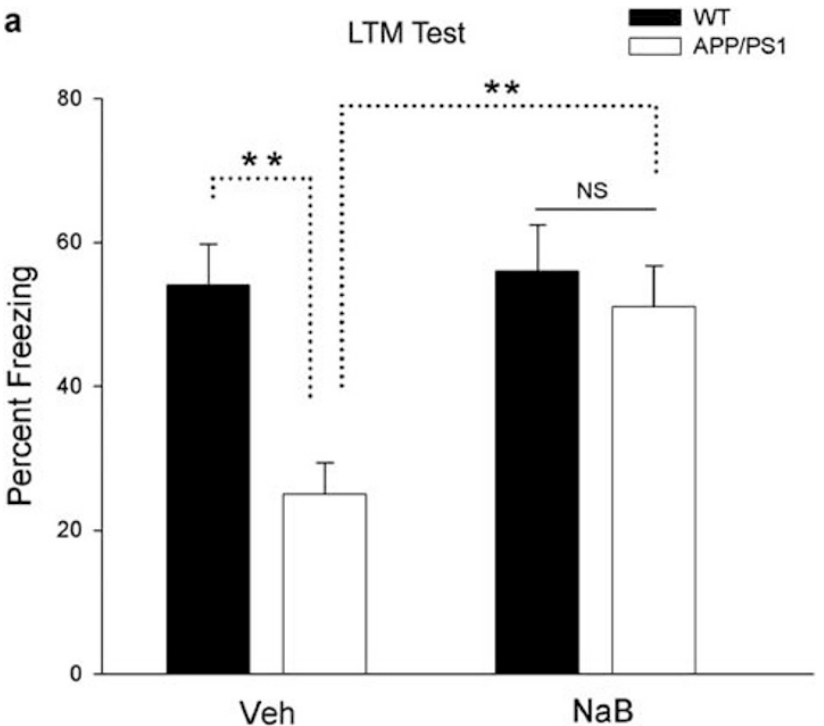

b

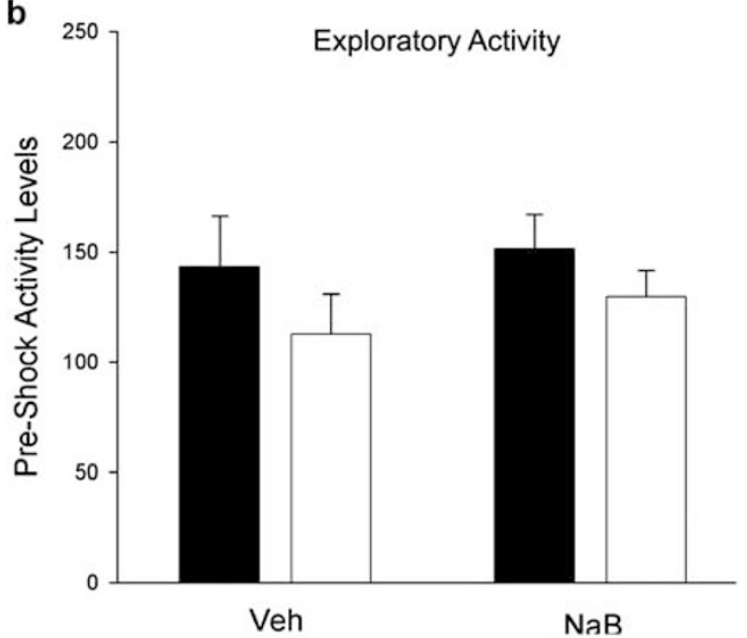

C

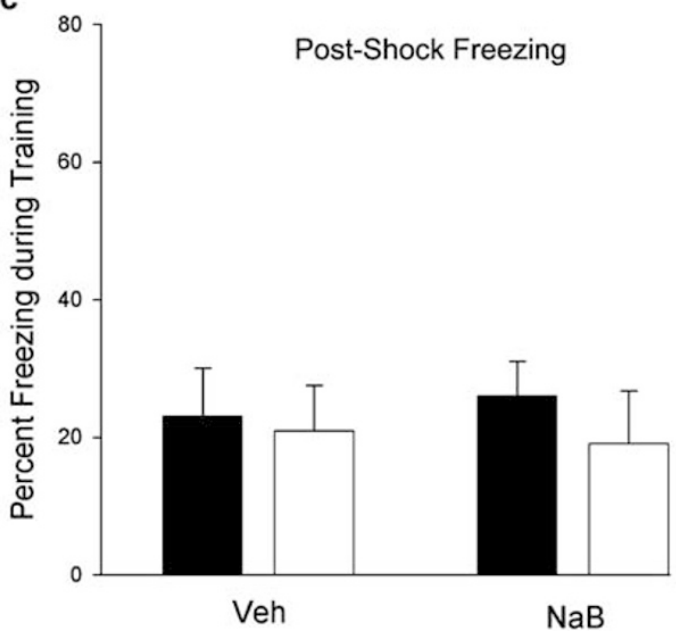

over time in the performance of the HDACi-treated animals or a decrease in the vehicle-treated groups. Therefore, we compared freezing responses between test days 1 and 15 within each group (Figure $5 \mathrm{~b}$ ). In both WT and APP/PS1 animals treated with vehicle, there was a significant reduction in freezing levels during the remote test relative to the recent test (Veh WT: $t_{(8)}=2.54, P<0.05$; Veh APP/ PS1: $\left.t_{(8)}=2.42, P<0.05\right)$. There was no such decline in freezing in either HDACi-treated group (NaB WT: $t_{(9)}=0.23, P>0.05 ; \mathrm{NaB}$ APP/PS1: $\left.t_{(9)}=0.35, P>0.05\right)$, indicating that the memory was better preserved in the WT and APP/PS1 animals receiving the HDACi. It is possible that extinction was affected by HDACi treatment, perhaps explaining the preservation of freezing between test days 1 and 15 in both WT and APP/PS1 mice. To address this possibility, we analyzed freezing at $1 \mathrm{~min}$ intervals during the $5 \mathrm{~min}$ expression test $24 \mathrm{~h}$ after training (Figure 5c). There was no difference in freezing among WT groups and the HDACi-treated APP/PS1 group at any point during this test $\left(P^{\prime} s>0.05\right)$, indicating that extinction learning was not altered by these compounds and was not a likely contributor to the preservation of freezing over the 2-week period.

We next isolated histone proteins from the hippocampus of WT and APP/PS1 mice and samples were subsequently immunoblotted for relative changes in histone acetylation. Interestingly, we did not detect significant differences in global $\mathrm{H} 3$ or $\mathrm{H} 4$ acetylation in the hippocampus when comparing genotypes in chronically injected mice $(\mathrm{H} 3$ : $\mathrm{F}_{(1,14)}=0.67, P>0.05, n=7$ (WT), $n=9$ (APP/PS1); H4: $\mathrm{F}_{(1,16)}=0.02, P>0.05, n=9(\mathrm{WT}), n=9$ (APP/PS1)). These data suggest that memory deficits in these mice are not caused by global abnormalities in histone acetylation. However, HDACi injections do cause transient elevations in histone acetylation in mice (Figure 6), as we detected significant $\mathrm{H} 4$ acetylation within $2 \mathrm{~h}$ of a single vorinostat injection $\left(\mathrm{F}_{(1,18)}=4.59, P<0.05\right)$. Therefore, when given daily for several weeks, these inhibitors cause repeated cycles of histone acetylation/deacetylation globally throughout the genome, providing a reasonable mechanism underlying the restoration of memory formation in this $\mathrm{AD}$ model.

Understanding the specific HDAC isoforms that are targeted by HDACi to enhance memory formation is important. This would provide not only insight into the underling molecular mechanisms involved in memory processes, but it may lead to the eventual design of more

Figure 4 Sodium butyrate $(\mathrm{NaB})$ rescues memory deficits in APPswe/ PSI IE9 mice. (a) Wild-type (WT) and APP/PSI (6-month-old) mice were injected with either vehicle (VEH) or $1.2 \mathrm{~g} / \mathrm{kg} \mathrm{NaB}$ for 21 days (VEH: WT $n=9$, APP/PSI $n=9 ; \mathrm{NaB}: \mathrm{WT} n=10$, APP/PS $\mid n=10$ ). All mice were then trained in a contextual fear conditioning paradigm and tested for longterm memory $24 \mathrm{~h}$ later. Freezing was measured for each animal. Two-way univariate analyses with Scheffe's post hoc tests were performed (Drug by Genotype: $F_{(3.34)}=4.81, P<0.05$; Veh WT vs Veh APP/PSI: $t_{(16)}=4.14$, $P<0.005$; Veh APP/PSI vs NaB APP/PSI: $\left.t_{(17)}=-3.57, P<0.005\right)$. $* * P<0.0$ I. (b) Exploratory activity was measured for each animal during $150 \mathrm{~s}$ period before the footshock. (Drug: $F_{(3,34)}=0.52$, Genotype: $F_{(3,34)}=0.47$, Drug by Genotype: $F_{(3,34)}=0.14 ; P>0.05$ for all). (c) Percent freezing was calculated for each animal during the $28 \mathrm{~s}$ period immediately following the footshock. (Drug: $F_{(3,34)}=0.01$, Genotype: $F_{(3,34)}=2.28$, Drug by Genotype: $F_{(3,34)}=0.07 ; P>0.05$ for all). 
selective and efficacious agents for the treatment of disorders involving cognitive deficits. However, to date there are conflicting reports on the isoform selectivity profile and potency of HDACi's. Previous studies have
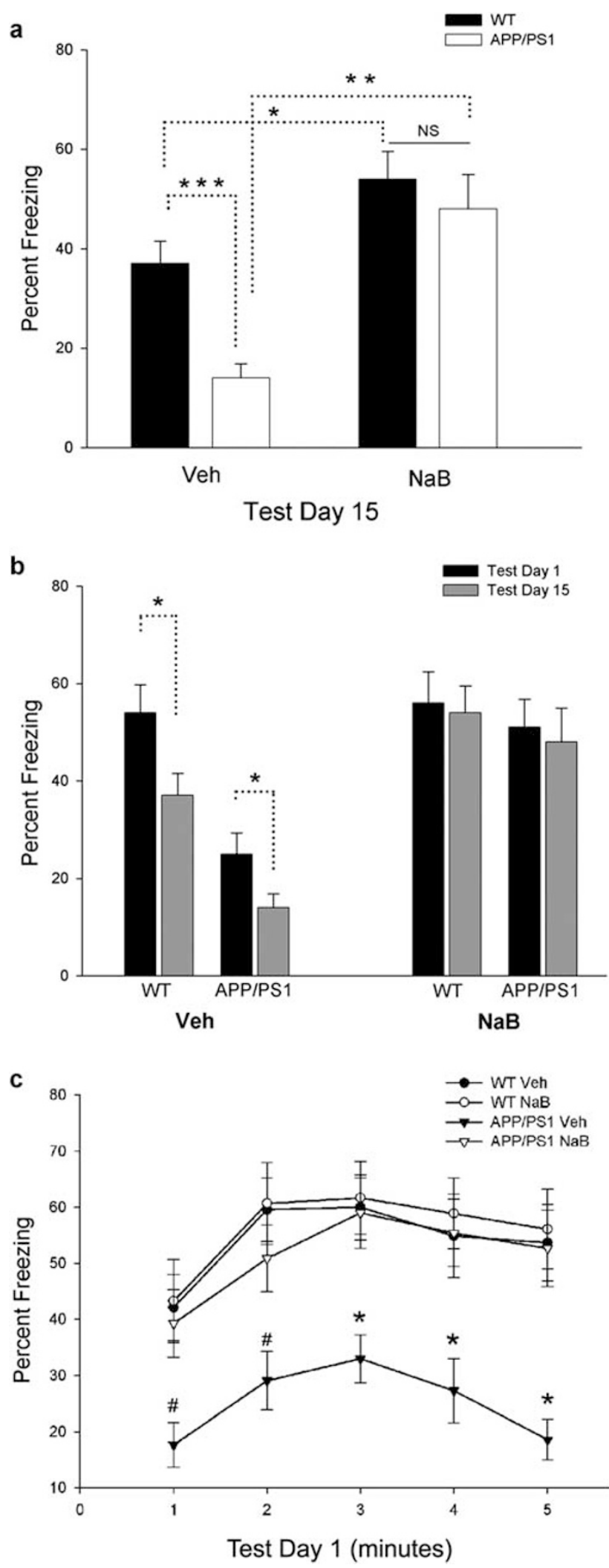

reported that VPA, butyrate, and SAHA have a pan-isoform profile with half-inhibitory concentration $\left(\mathrm{IC}_{50}\right)$ values ranging from low micromolar to millimolar ranges. However, it has become clear recently that immunoprecipitated and even recombinantly expressed and purified class IIa HDACs (eg, HDAC4) associate with class I HDACs and that these co-purifying enzymatic activities can occlude the true selectivity and potency of inhibitor treatments (Lahm et al, 2007; Jones et al, 2008).

To overcome these limitations, we have implemented biochemical assays of the deacetylase activity of recombinant human HDAC1-9 expressed and purified from Sf9 insect cells. These assays rely on fluorogenic class I and class II HDAC substrates that show an improved ability in the latter case to specifically detect deacetylase activity from class II HDAC isoforms free of contribution of any copurifying class I HDAC activity that otherwise confounds the data interpretation (Bradner et al, 2009). As summarized in Table 1, using these assays in a kinetic mode under conditions where appropriate substrates for each isoform are used at their $K_{\mathrm{m}}$ and the rate of the reaction is monitored over time, VPA (Supplementary Figure 1), NaB (Supplementary Figure 2), and phenylbutyrate (data not shown) were found to have $\mathrm{IC}_{50}$ 's in the low micromolar range against $\mathrm{HDAC} 1-3$ and 8 with effectively no inhibition against HDAC4-7 and HDAC9. In contrast, and similar to

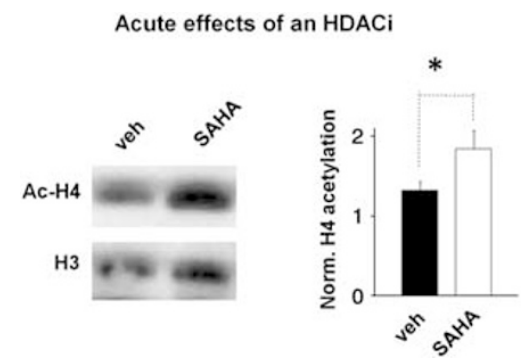

Figure $\mathbf{6}$ Systemic injections of inhibitors of histone deacetylases (HDACi's) cause elevations in global histone acetylation. Wild-type C57/ Bl6 mice were injected once with vehicle $(n=5)$ or $50 \mathrm{mg} / \mathrm{kg}$ suberoylanilide hydroxamic acid (SAHA, $n=5)$ and then killed $2 \mathrm{~h}$ later. Purified histone proteins from the hippocampus were immunoblotted for acetylated $\mathrm{H} 4(\mathrm{Ac}-\mathrm{H} 4)$ and total $\mathrm{H} 3(\mathrm{H} 3$; loading control). Normalized H4 acetylation was calculated by dividing the integrated density (ID) of Ac-H4 by the ID of $\mathrm{H} 3$ for each sample. One-way analysis of variance (ANOVA, $* P<0.05)$.

Figure 5 Sodium butyrate $(\mathrm{NaB})$ treatment stabilizes newly consolidated memories in APPswe/PSIdE9 mutants. (a) Mice trained and tested (Figure 4 ) continued to receive daily vehicle or $\mathrm{NaB}$ for an additional 14 days. All mice were then reexposed to the conditioning context to reactivate the memory. Two-way univariate analyses with Scheffe's post hoc tests were performed. $* P<0.05$, **P $<0.01$, **** $P<0.00$ I. (b) Freezing performance from the first (test day I, $24 \mathrm{~h}$ after training) and second (test day 15, 2 weeks after training) retrieval tests are directly compared for each drug treatment and genotype. Paired samples $t$-tests were performed. $* P<0.05$. (c) Freezing behavior during the 5 min retrieval test given $24 \mathrm{~h}$ after training (Figure 4a) was analyzed in I min intervals to visualize the rate, if any, of extinction in each group. As reported, there was no difference at any time point between WT and APP/PSI mice treated with NaB. APP/ PSI mice treated with Veh were, however, different from WT mice at the first two time points $\left({ }^{\#} P<0.05\right)$ and different from all groups during minutes $3-5(* P<0.05)$. 
Table I IC 50 Values of HDAC Inhibitors that Increase Memory Formation in APPswe/PSI dE9 Mutant Mice

\begin{tabular}{|c|c|c|c|c|c|}
\hline Compound & \multicolumn{5}{|l|}{ Class I } \\
\hline Valproate & $35.5 \pm 3.1$ & $59.3 \pm 3.6$ & $218.5 \pm 22.5$ & $97.1 \pm 5.8$ & \\
\hline SAHA & $0.002 \pm 0.0001$ & $0.003 \pm 0.0001$ & $0.006 \pm 0.0003$ & $0.7 \pm 0.07$ & \\
\hline Compound & HDAC4 & HDAC5 & HDAC7 & HDAC9 & HDAC6 \\
\hline Butyric acid & $5725 \pm 98.3$ & $6403 \pm 370$ & $4380 \pm 441$ & $5614 \pm 359$ & $588| \pm| 303$ \\
\hline Valproate & $>15000$ & $>15000$ & $>15000$ & $>15000$ & $>20000$ \\
\hline SAHA & $>25$ & $>15$ & $>25$ & $>25$ & $0.004 \pm 0.0004$ \\
\hline
\end{tabular}

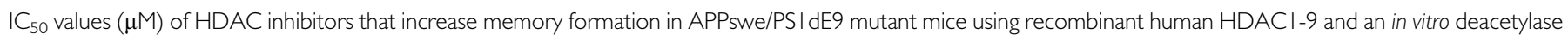

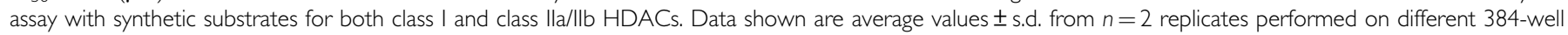
plates.

what has been reported previously in the literature, SAHA (Supplementary Figure 3) showed nanomolar potency toward HDAC1-3 and 6 and a mild inhibition of HDAC8 while having $\mathrm{IC}_{50}$ values $>10 \mu \mathrm{M}$ for HDAC4-7 and HDAC9. Thus, VPA, NaB, phenylbutyrate, and SAHA share in common the inhibition of the class I HDACs1-3, suggesting that in vivo the activity of all three, or a subset, of these HDAC family isoforms may be responsible for modulating memory formation. These data also explain the shared efficacy of these three distinct compounds regarding rescue of cognitive deficits in this $\mathrm{AD}$ model.

\section{DISCUSSION}

The key finding in our study is that three distinct inhibitors of class I HDACs completely rescue memory deficits in a mouse model of AD. Importantly, SAHA, which is FDA approved for the treatment of cancer under the trade name Zolinza $\AA$, was effective in restoring memory performance in this AD model. We believe that HDACi's rescue freezing deficits in APP/PS1 mice by improving memory formation. After chronic HDACi treatments, the fear memory of mutants is indistinguishable from WT mice. Importantly, HDACi treatments do not affect other measures of behavior in these tasks, including exploratory activity and immediate freezing. Thus, the observed rescue of freezing in response to exposure to the associative stimulus cannot be explained by state-dependent effects, such as reduced locomotion. Therefore, our data suggest that HDACi's rescue freezing deficits in the APP/PS1 mice by promoting memory formation through enhancing consolidation or by rescuing a retrieval deficit. This exciting finding provides important preclinical evidence for the hypothesis that HDACi's may improve cognitive function in AD patients.

We hypothesize that HDACi's promote memory formation in this $\mathrm{AD}$ model by enhancing histone acetylation. The mammalian genome encodes for 18 HDAC isoforms, which can be divided into four distinct classes (I-IV) based on a variety of factors, including catalytic mechanism, cofactors, patterns of expression, and sensitivity to various classes of inhibitors (Haberland et al, 2009). Although the specific isoforms involved in memory and other neurological processes are poorly understood at this point, very recent work has begun the task of defining their functional roles in the CNS. For example, HDAC1 and 2, which are class I HDACs and nearly identical, are required for normal neuronal differentiation during development (Montgomery et al, 2009). Interestingly, Tsai and colleagues showed an additional key function for HDAC2 as a negative regulator of synaptic plasticity and memory formation in the adult CNS (Guan et al, 2009). In addition, the emotional salience of stimuli such as stress or drugs of abuse is regulated by activity of the class II HDAC5 within the nucleus accumbens, the brain's reward center (Renthal et al, 2007). In this study, we used contextual fear conditioning, a hippocampus-dependent task. Several HDAC isoforms are highly expressed in this brain regions (Broide et al, 2007). Using synthetic substrates that enable one to distinguish between class I and class II HDAC activities (Jones et al, 2008; SJ Haggarty, Personal Communication), we found that the HDACi's used in this study predominantly target the deacetylase activity of class I HDAC isoforms (HDAC1, 2, 3,8 ), with HDAC6, a class IIb isoform, also being targeted by SAHA (Table 1). Thus, although we cannot determine which isoform(s) is critical for amelioration of the memory deficit or its subsequent maintenance from our current data, our findings are consistent with those of Guan et al (2009) in terms of supporting an important role for class I HDAC isoforms. As isoform-selective inhibitors are a vigorous area of development in cancer therapeutics, identifying the isoform(s) critical to our effects will likely provide potent, druggable targets for therapeutics aimed at the cognitive deficits associated with $\mathrm{AD}$.

Our behavioral results are consistent with a recent study showing improvement of APPswe transgenic mice in a spatial learning task after repeated injections of the HDACi, phenylbutyrate (Ricobaraza et al, 2009). Our HDAC biochemical assay indicates that phenylbutryate shares an HDAC isoform profile similar to butyrate and VPA. In contrast to our findings in 6-month-old APP/PS1 mice, the 
authors observed a significant reduction in $\mathrm{H} 4$ acetylation in much older mice from this distinct transgenic line. The most parsimonious explanation for these contrasting results is that the studies used distinct mouse models at different ages. We used an aggressive model of familial AD for our studies, where plaque deposits appear between 5 and 6 months of age (Jankowsky et al, 2004; Jankowsky et al, 2005). In contrast, Ricobaraza et al (2009) used the TG2567 line that does not begin to develop plaque deposits or cognitive anomalies until 1 year of age (Gotz et al, 2004). Indeed, their study reported a deficit in $\mathrm{H} 4$ acetylation in animals that were 18 months of age (Ricobaraza et al, 2009). Therefore, it is possible that a global decrease in histone acetylation is promoted by a combination of advanced age and elevated levels of $\mathrm{A} \beta$. In addition, we did not observe effects of $\mathrm{NaB}$ on freezing in WT mice at any age tested. This finding contrasts with a recent study that used a similar dosing schedule of $\mathrm{NaB}$ and contextual fear conditioning (Guan et al, 2009). There are several possible explanations for these discordant results. Our WT mice were much older and had a different genetic background than the mice used in the study by Guan et al. In addition, the fear conditioning protocol used in Guan et al. study resulted in very low freezing levels in saline-treated mice. Thus, in addition to genetic background differences that may impact the acquisition, encoding, or expression of conditioned fear, we speculate that $\mathrm{NaB}$ enhances contextual freezing in young mice presented with weak context-shock associations, but not in older mice that receive stronger training.

From a memory systems standpoint, we envision several processes that could be affected by chronic HDACi treatment. First, HDACi's may facilitate cognitive function in this model through broad network (ie, system) effects, possibly by promoting dendritic growth and synaptogenesis (Fischer et al, 2007; Guan et al, 2009). This would allow for alternate strategies to consolidate and/or retrieve information through enhanced neural connectivity (Buckner, 2004; Jankowsky et al, 2005). Another possibility may involve the ability of particular HDAC isoforms to deacetylate proteins in addition to histones, such as transcription factors (Yeh et al, 2004). Finally, this method could enhance memory consolidation by altering the cellular processes that support information storage. This hypothesis is distinct from the others, as it relates specifically to the cell and molecular processes that are believed to contribute to memory formation in the hippocampus. For instance, transcription of memory-promoting genes could be primed by HDACi treatments, essentially increasing the 'gain' of the molecular machinery that supports memory formation. In support of this idea, we show that a single dose of $\mathrm{NaB}$ results in a transient elevation of histone acetylation, though we did not detect a global elevation in histone acetylation after 3 weeks of $\mathrm{NaB}$ treatments. Thus, repeated, systematic HDACi dosing may have unique effects on gene activation resulting from the phasic regulation of global histone acetylation. This type of chronic treatment likely results in downstream biochemical effects that are distinct from those of a single dose of an HDACi. Indeed, post-training infusions of the HDACi trichostatin A enhance contextual fear conditioning and histone acetylation in WT mice (Vecsey et al, 2007). The absence of a similar memory enhancement in our WT mice after HDACi treatment may be due to differences in the specific compound used, how it was delivered and how many times. For example, direct infusion of HDACi's into the hippocampus may regulate a subset of genes distinct from those targeted by repeated systemic injections. This distinction should not be underestimated. In contrast to acute delivery of HDACi directly into the hippocampus of $\mathrm{AD}$ mice (Francis et al, 2009), the chronic treatment used in this study likely causes a repeated cycling of histone acetylation and deacetylation. Importantly, this chronic dosing is likely analogous to the therapeutic treatment regimen that would be used in patients with AD. Future studies will hopefully assess the gene- and brain-regionspecific effects of systemic $v s$ acute class I HDAC inhibition in both normal and AD mice.

Our remote memory experiment revealed an additional positive effect of HDACi's on contextual memory. As expected, we observed memory deficits between 1- and 15-day retrieval tests in both genotypes treated with saline. In contrast, HDACi-treated mice, regardless of genotype, showed no sign of reduced memory between retrieval tests. These data suggest that HDACi's protect previously consolidated memories from weakening in response to time and/or reactivation. Several possibilities could account for the contextual freezing differences observed in salinetreated mice. Extinction of fear is one possible explanation. However, because fear memory was preserved in HDACitreated mice, this would require that HDACi's negatively regulate extinction learning. Such an event is unlikely, given that HDACi's have recently been shown to enhance extinction of contextual memories (Bredy and Barad, 2008; Lattal et al, 2007). In addition, we did not observe a difference in extinction learning among groups in our studies. Therefore, we believe that the HDACi's are more likely to be regulating another aspect of memory stability; reconsolidation, maintenance or retrieval. It is possible that the process of reconsolidation is not entirely efficient (Bredy and Barad, 2008). This could result in a slight weakening of the memory following multiple retrieval tests. HDACi's may, therefore, improve the efficiency of reconsolidation. Alternatively, the 'HDACi-protected' memory could relate to the growing involvement of the cortex in system consolidation. The role of cortical association areas in contextual fear memories develops over the course of 2 weeks (Frankland et al, 2004; Matynia et al, 2008; Maviel et al, 2004), and synaptic plasticity in cortical circuits is believed to be an important contributor to this process (Frankland et al, 2001). Freezing responses may become less robust as fear memories are distributed across multiple networks. In this scenario, HDACi's could act to facilitate 'uploading' of contextual information to other brain areas, perhaps by enhancing cortical synaptic plasticity. Indeed, HDACi's have been shown to facilitate long-term potentiation at hippocampal synapses (Levenson et al, 2004; Guan et al, 2009), though it is currently not known if these compounds have similar effects on synaptic plasticity in cortical circuits. Finally, the memory deficits observed in vehicle-treated mice could be explained by the relative difficulty of retrieving a remote memory. If a stabilized memory is more difficult than a recent memory to retrieve, then HDACi's may facilitate this aspect of cognition. Given the obvious therapeutic applications for cognitive 
improvement, it will be very interesting to further explore the effects of HDACi's on remote memory processes.

In summary, our study provides evidence that several distinct HDACi's rescue cognitive deficits in a genetically engineered mouse model of AD. At present, a variety of specific and potent HDACi's are under development and clinical testing. Our data strongly support the idea that facilitating histone acetylation through systemic delivery of HDACi's targeting class I HDAC's is a promising new avenue for the treatment of cognitive abnormalities associated with $\mathrm{AD}$.

\section{ACKNOWLEDGEMENTS}

We thank Felecia Hester and Ivonne Rivera for technical assistance. The original breeding pairs of the APP/PS1 mouse line were generously provided by $\mathrm{Dr}$ Ling Li. This work was supported by the NIMH, NINDS, American Health Assistance Foundation, The Rotary CWSS International, Philip Morris External Research Program, and the Evelyn F McKnight Brain Research Foundation. SJH, DMF, and $\mathrm{KMH}$ were supported by the Stanley Medical Research Institute and NARSAD. Dr Ralph Mazitschek generously provided MAZ1600 and MAZ1675 HDAC substrates. We also thank Erik Roberson and others at the UAB Alzheimer's Disease Research Center (ADRC) for critical evaluation of this paper.

\section{DISCLOSURE}

JDS is a consultant for EvVivo Pharmaceuticals. Except as noted in the acknowledgment, all other authors declare that, except for income received from primary employer, no financial support or compensation has been received from any individual or corporate entity over the past three years for research or professional service and there are no personal financial holdings that could be perceived as constituting a potential conflict of interest.

\section{REFERENCES}

Alarcon JM, Malleret G, Touzani K, Vronskaya S, Ishii S, Kandel ER et al (2004). Chromatin acetylation, memory, and LTP are impaired in $\mathrm{CBP}+/-$ mice: a model for the cognitive deficit in Rubinstein-Taybi syndrome and its amelioration. Neuron 42: 947-959.

American Psychiatric Association. Task Force on DSM-IV (1994). Diagnostic and Statistical Manual of Mental Disorders: DSM-IV, 4th edn. American Psychiatric Association: Washington, DC. xxvii, 886p.

Berger SL (2007). The complex language of chromatin regulation during transcription. Nature 447: 407-412.

Braak H, Braak E (1998a). Evolution of neuronal changes in the course of Alzheimer's disease. J Neural Transm Suppl 53: 127-140.

Braak H, Braak E, Bohl J, Bratzke H (1998b). Evolution of Alzheimer's disease related cortical lesions. J Neural Transm Suppl 54: 97-106.

Bredy TW, Barad M (2008). The histone deacetylase inhibitor valproic acid enhances acquisition, extinction, and reconsolidation of conditioned fear. Learn Mem 15: 39-45.
Broide RS, Redwine JM, Aftahi N, Young W, Bloom FE, Winrow CJ (2007). Distribution of histone deacetylases 1-11 in the rat brain. J Mol Neurosci 31: 47-58.

Buckner RL (2004). Memory and executive function in aging and AD: multiple factors that cause decline and reserve factors that compensate. Neuron 44: 195-208.

Dickson DW (1997). Neuropathological diagnosis of Alzheimer's disease: a perspective from longitudinal clinicopathological studies. Neurobiol Aging 18(4 Suppl): S21-S26.

Dudai Y (2004). The neurobiology of consolidations, or, how stable is the engram? Annu Rev Psychol 55: 51-86.

Eichenbaum H (2000). A cortical-hippocampal system for declarative memory. Nat Rev Neurosci 1: 41-50.

Fischer A, Sananbenesi F, Wang X, Dobbin M, Tsai LH (2007). Recovery of learning and memory is associated with chromatin remodelling. Nature 447: 178-182.

Francis YI, Fa M, Ashraf H, Zhang H, Staniszewski A, Latchamn DS et al (2009). Dysregulation of histone acetylation in the APP/PS1 mouse model of Alzheimer's disease. J Alzheimers Dis 18: 131-139.

Frankland PW, Bontempi B (2005). The organization of recent and remote memories. Nat Rev Neurosci 6: 119-130.

Frankland PW, Bontempi B, Talton LE, Kaczmarek L, Silva AJ (2004). The involvement of the anterior cingulate cortex in remote contextual fear memory. Science 304: 881-883.

Frankland PW, O'Brien C, Ohno M, Kirkwood A, Silva AJ (2001). Alpha-CaMKII-dependent plasticity in the cortex is required for permanent memory. Nature 411: 309-313.

Gediya LK, Chopra P, Purushottamachar P, Maheshwari N, Njar VC (2005). A new simple and high-yield synthesis of suberoylanilide hydroxamic acid and its inhibitory effect alone or in combination with retinoids on proliferation of human prostate cancer cells. J Med Chem 48: 5047-5051.

Gotz J, Streffer JR, David D, Schild A, Hoerndli F, Pennanen L et al (2004). Transgenic animal models of Alzheimer's disease and related disorders: histopathology, behavior and therapy. Mol Psychiatry 9: 664-683.

Guan JS, Haggarty SJ, Giacometti E, Dannenberg JH, Joseph N, Gao J et al (2009). HDAC2 negatively regulates memory formation and synaptic plasticity. Nature 459: 55-60.

Haass C, Selkoe DJ (2007). Soluble protein oligomers in neurodegeneration: lessons from the Alzheimer's amyloid betapeptide. Nat Rev Mol Cell Biol 8: 101-112.

Haberland M, Montgomery RL, Olson EN (2009). The many roles of histone deacetylases in development and physiology: implications for disease and therapy. Nat Rev Genet 10: 32-42.

Jankowsky JL, Fadale DJ, Anderson J, Xu GM, Gonzales V, Jenkins NA et al (2004). Mutant presenilins specifically elevate the levels of the 42 residue beta-amyloid peptide in vivo: evidence for augmentation of a 42-specific gamma secretase. Hum Mol Genet 13: 159-170.

Jankowsky JL, Melnikova T, Fadale DJ, Xu GM, Slunt HH, Gonzales $\mathrm{V}$ et al (2005). Environmental enrichment mitigates cognitive deficits in a mouse model of Alzheimer's disease. J Neurosci 25: 5217-5224.

Jenuwein T, Allis CD (2001). Translating the histone code. Science 293: $1074-1080$.

Jones P, Altamura S, De Francesco R, Gallinari P, Lahm A, Neddermann $P$ et al (2008). Probing the elusive catalytic activity of vertebrate class IIa histone deacetylases. Bioorg Med Chem Lett 18: 1814-1819.

Kandel ER (2001). The molecular biology of memory storage: a dialog between genes and synapses. Biosci Rep 21: 565-611.

Korzus E, Rosenfeld MG, Mayford M (2004). CBP histone acetyltransferase activity is a critical component of memory consolidation. Neuron 42: 961-972.

Kouzarides T (2007). Chromatin modifications and their function. Cell 128: 693-705. 
Epigenetic memory rescue in a model of Alzheimer's M Kilgore et al

Lahm A, Paolini C, Pallaoro M, Nardi MC, Jones P, Neddermann P et al (2007). Unraveling the hidden catalytic activity of vertebrate class IIa histone deacetylases. Proc Natl Acad Sci USA 104: $17335-17340$.

Lambert MP, Barlow AK, Chromy BA, Edwards C, Freed R, Liosatos $M$ et al (1998). Diffusible, nonfibrillar ligands derived from Abeta1-42 are potent central nervous system neurotoxins. Proc Natl Acad Sci USA 95: 6448-6453.

Lattal KM, Barrett RM, Wood MA (2007). Systemic or intrahippocampal delivery of histone deacetylase inhibitors facilitates fear extinction. Behav Neurosci 121: 1125-1131.

Levenson JM, O'Riordan KJ, Brown KD, Trinh MA, Molfese DL, Sweatt JD (2004). Regulation of histone acetylation during memory formation in the hippocampus. J Biol Chem 279: 40545-40559.

Matynia A, Anagnostaras SG, Wiltgen BJ, Lacuesta M, Fanselow MS, Silva AJ (2008). A high through-put reverse genetic screen identifies two genes involved in remote memory in mice. PLoS ONE 3: e2121.

Maviel T, Durkin TP, Menzaghi F, Bontempi B (2004). Sites of neocortical reorganization critical for remote spatial memory. Science 305: 96-99.

McGaugh JL (2000). Memory - a century of consolidation. Science 287: 248-251.

Medina JH, Bekinschtein P, Cammarota M, Izquierdo I (2008). Do memories consolidate to persist or do they persist to consolidate? Behav Brain Res 192: 61-69.

Montgomery RL, Hsieh J, Barbosa AC, Richardson JA, Olson EN (2009). Histone deacetylases 1 and 2 control the progression of neural precursors to neurons during brain development. Proc Natl Acad Sci USA 106: 7876-7881.

Phillips RG, LeDoux JE (1992). Differential contribution of amygdala and hippocampus to cued and contextual fear conditioning. Behav Neurosci 106: 274-285.
Renthal W, Maze I, Krishnan V, Covington 3rd HE, Xiao G, Kumar A et al (2007). Histone deacetylase 5 epigenetically controls behavioral adaptations to chronic emotional stimuli. Neuron 56: 517-529.

Ricobaraza A, Cuadrado-Tejedor M, Perez-Mediavilla A, Frechilla D, Del Rio J, Garcia-Osta A (2009). Phenylbutyrate ameliorates cognitive deficit and reduces tau pathology in an Alzheimer's disease mouse model. Neuropsychopharmacology 34: 1721-1732.

Selkoe DJ (2001). Alzheimer's disease: genes, proteins, and therapy. Physiol Rev 81: 741-766.

Squire LR, Stark CE, Clark RE (2004). The medial temporal lobe. Annu Rev Neurosci 27: 279-306.

Vecsey CG, Hawk JD, Lattal KM, Stein JM, Fabian SA, Attner MA et al (2007). Histone deacetylase inhibitors enhance memory and synaptic plasticity via CREB:CBP-dependent transcriptional activation. J Neurosci 27: 6128-6140.

Walsh DM, Klyubin I, Fadeeva JV, Cullen WK, Anwyl R, Wolfe MS et al (2002). Naturally secreted oligomers of amyloid beta protein potently inhibit hippocampal long-term potentiation in vivo. Nature 416: 535-539.

Wong PC, Cai H, Borchelt DR, Price DL (2002). Genetically engineered mouse models of neurodegenerative diseases. Nat Neurosci 5: 633-639.

Wood MA, Attner MA, Oliveira AM, Brindle PK, Abel T (2006). A transcription factor-binding domain of the coactivator CBP is essential for long-term memory and the expression of specific target genes. Learn Mem 13: 609-617.

Yeh SH, Lin CH, Gean PW (2004). Acetylation of nuclear factor-kappaB in rat amygdala improves long term but not short-term retention of fear memory. Mol Pharmacol 65: 1286-1292.

Supplementary Information accompanies the paper on the Neuropsychopharmacology website (http://www.nature.com/npp) 